\title{
Synthetic Characters with Emotional States
}

\author{
Nikos Avradinis, Themis Panayiotopoulos, Spyros Vosinakis \\ Knowledge Engineering Lab, University of Piraeus, Department of Informatics, \\ 80, Karaoli \& Dimitriou Street, 185 34, Piraeus, Greece, \\ \{avrad, themisp, spyrosv\}@unipi.gr
}

\begin{abstract}
A new trend that has emerged in the field of applied artificial intelligence in the past few years is the incorporation of emotion emulation mechanisms in situated agent systems. Several researchers in the field of psychology and neural science agree that emotion is an essential aspect of human intelligence, diverging from the traditional treatment of emotion as something that inhibits rational thinking. This argument has also influenced the area of artificial intelligence and especially the young, yet vibrant field of Intelligent Virtual Agents, where it has become accepted that emotion is a key issue to achieve believable agent behaviour. The increased interest for emotions has resulted in several computational emotional models having been presented, with diverse approaches, generically classified into cogn itive and non-cognitive, inspired by areas such as neural science. Hybrid approaches have also appeared, combining both cognitive and noncognitive elements in an attempt to accurately describe the inner workings of emotional mechanisms. Such a hybrid model is the one we adopt in this paper, aiming to apply it to SimHuman, an intelligent virtual agent platform, in order to introduce emotion awareness and affective behaviour to the agent architecture.
\end{abstract}

\section{Introduction}

Emotion has been traditionally treated as a factor that inhibits rational thinking. Considered as a disorganized human response, emotion was labelled inappropriate for decision-making, and although it might be suitable for art or entertainment, it had better be kept out of scientific work.

This long-standing view on emotions has lately been strongly questioned. Researchers, mainly from the area of psychology and neural science, agree that emotion is an essential aspect of human intelligence. Particularly influential towards establishing this notion were two popular works by Le Doux and Damasio in the mid-1990's [1,2].

Long before Damasio's and Le Doux's works, various researchers had argued about the importance of emotions $[3,4,5]$. However, conclusions drawn from research in neurology and psychology started having a stronger influence in the early nineties, when several works on intelligent agents adopting ideas from the field of psychology were presented $[6,7,8,9]$. The A I research community started 
showing an increased interest for the incorporation of emotion-handling mechanisms into embodied intelligent agent systems, which had as a result the evolution of emotional agents into a new, highly active research field. The importance of emotions became even more apparent with the appearance of virtual agent systems, as they provided a basic feature classic agent systems lacked-embodiment. By assuming the existence of a virtual body, agent systems moved from merely textual forms of communication to highly expressive non-verbal communication means, such as body posture or eye gaze [10]. These new ways of communication were particularly suitable to express emotions, which provided a strong motivation to continue and expand research work in this field.

\section{Defining emotions}

While there is a lot of talk going on about emotions, a universal and authoritative definition is difficult to be given. Many attempts to define emotion have been made, with more than ninety of them having been reviewed in [11]. In the same paper, Kleinginna and Kleinginna propose what is considered one of the most comprehensive definitions of emotions:

"Emotion is a complex set of interactions among subjective and objective factors, mediated by neural/hormonal systems, which can:

- (a) give rise to affective experiences such as feelings of arousal, pleasure/displeasure;

- (b) generate cognitive processes such as emotionally relevant perceptual fffects, appraisals, labeling processes;

- (c)activate widespread physiological adjustments to the arousing conditions; and

- (d) lead to behavior that is often, but not always, expressive, goal directed, and adaptive".

The above approach defines emotions as relevant with cognitive as well as subcognitive processes, placing subcognitive systems at an entry level, driving the higher level subsystems. Although including goal-oriented behaviour, the definition differentiates from other approaches such as Oatley's one [12], where goal management is considered an essential attribute of emo tions.

A lot of the confusion about emotions is attributed to the fact that, being a common word in every day language, the term is used to describe various closely related, yet not identical concepts, such as moods, drives or emotional states. As a general rule, emotions are responses to environmental input that produce an intense short-term affective state-an emotional state [8]. So, if one experiences fear because of a threatening event, he is afraid. A mood, on the other hand, is a longer-term affective state, that cannot be clearly attributed to a specific cause-for example, depression, in its common rather than its clinical definition can be considered a mood. Drives are mainly physical and although not emotions themselves, they can activate or influence affective processes -for example, hunger can make somebody irritated. 


\section{Do agent systems really need emotions?}

One could understandably pose the question "Why should I bother to put emotions in an agent system?. After all, I want my computer to be able to assist my work in an efficient way-I don't need an agent greeting me with a smile every time I switch it on or start nagging whenever I make a mistake".

This can be easily answered when one considers applications involving some sort of social interaction among agent and environment, agent and human user or agents among themselves. When social issues emerge, purely rational agents prove insufficient, as the focus is not on providing the best solution to a welldefined problem, but rather producing an output that is suitable and in context. Applications like interactive storytelling [13], education, training in social and everyday skills [14] are exa mples where awareness on emotions is essential.

The need for mechanisms that can model and handle emotions is even more apparent in applications that include naturalistic animated visual representations of the agent, where the issue of believability comes to surface. Even the simplest forms of such applications, like two-dimensional talking heads, require attention so that the agent's representation does not seem robotic and lifeless. Animation sequences should be coherent with the action the agent is performing or the message it is trying to communicate. A virtual newscaster, for example, would seem absolutely fake if it was presenting breaking news about a tragic accident with a bright, smi ling face. Being able to assume a suitable facial expression requires emotion-understanding skills from the part of the agent system, so that it can recognize the emotional impact of the news it is attempting to present.

The importance of emotions is even more apparent in Virtual Reality applications where full-scale agent embodiment raises believability standards even higher. Providing multi-modal means of communication, 3D models of agents situated in virtual environments are expected by the user not only to look realistic, but, more important, demonstrate believable reactions and behaviour, consistent with the states they are in, their own internal attributes as well as the stimuli they receive.

\section{Physical aspects of emotion in synthetic agents}

Having established the value of incorporating emotions into software agent applications, one has to consider what characteristics an agent should have in order to be considered "emotionally aware". A first look into the issue reveals two aspects of it: Emotion Recognition and Emotion Expression.

Emotion recognition implies the ability to guess the emotional state of the agent itself, as well as other agents situated in the environment either human or synthetic. Recognition can be performed by observing a wide range of signals as well as reasoning about situations that generate emotions. A list of such signals is presented below, based on a list of sentic modulations presented by Picard in [8], The list refers to humans and is classified into two categories according to how easily these signs can be perceived by an external observer. 
Physical Signs of Emotions

\begin{tabular}{l|l}
\hline Easy to perceive & Difficult to percei ve \\
\hline Facial expression & Respiration rate \\
Voice intonation & Heart/pulse rate \\
Gestures & Temperature \\
Movement & Perspiration \\
Posture & Muscle \\
Eye gaze & Blood Pressure \\
& Body Odour
\end{tabular}

In order to recognise emotional states, an agent should be able to perceive the above signs in addition to verbal emotional expressions and reason about them in context with the environment. However, just being able to recognise emotions is not enough for an emotional agent. It should also be able to express emotions in a way perceptible by other agents, which means that at a physical level it should be able to produce signs such as the above.

\section{Expressing emotions in virtual agents}

Not all of the above features are easy or even feasible to be modelled and communicated with existing technology. However, most current virtual agent systems support all or some of the following:

- Facial expressions

- Movement

- Gestures

- Head orientation \& Eye gaze

- Posture

- Vocal intonation

- Linguistic expression

- Odour emission

It is easily understood that emotion expression is finally a compound product, emerging as a resultant of the synthesis of more than one of the above expressive means. Expressing an emotion can either be a deliberate and conscious act, like a willingly generated smile, or a spontaneous one, as a result of a certain emotional state, like a shaking hand because of nervousness.

\section{Emotion generation in synthetic agents}

Assuming a synthetic agent system incorporates all necessary means of emotion recognition and expression, there is still something missing so that it can be 
characterized as a full scale emotional agent. This is a mechanism to handle and generate emotions in a believable way, consistent with the input received from the environment. This mechanism should interact with the decision-making and motor components of the agent, so that emotional effects on the agent's actions can be taken into account and properly manipulated. In [8] the basic characteristics of an emotional computer-based system is presented, summarized in the five generic principles shown below:

1. The system should demonstrate behaviour that appears to an external observer as a result of emotional processes

2. The system should have spontaneous, low level emotional responses to certain stimuli

3. The system should be able to cognitively process and generate emotions by reasoning about situations, especially when these concern its goals, standards, preferences and expectations in some way.

4. The system should be able to have an emotional experience and be aware of its cognitive, physiological and subjective feeling aspects.

5. The system's emotions should interact with other processes that imitate human functions, including cognitive ones such as memory, perception, decision making, leaming, but also physical ones, such as sentic modulations.

Most of the emotional agent implementations presented up to date incorporate some sort of mechanism that partially supports the above principles. In an attempt to produce more realistic emotional responses, the AI community has turned towards the field of psychology and neuroscience, adopting theories and models devised by researchers in these areas. Custom emotional models are not uncommon, but the majority of them are in some way or another based on or influenced by es tablished emotion theories.

\section{Models of emotion}

Theories about emotion generation are not a new development in science. Philosophical approaches concerning emotion first appeared in ancient times, with more comprehensive theories based on scientific observation rather than philosophy becoming available in the $19^{\text {th }}$ century, such as Darwin's research on animal and human emotional expressions. However, the majority of the modern emotional theories are dated to the 1960's and forth.

The numerous different approaches presented so far can be roughly classified into two broad categories-cognitive and non-cognitive theories. The distinction between cognitive and sub-cognitive is directly analogous to the brain and body separation, and is part of a long-standing debate on whether emotions are cognitive, therefore a mental process or physical, therefore a bodily process.

The former approach is a high-level one, treating emotions as a result of symbolic, cognitive processing that involves reasoning. Examples of this category are Frijda's [15] and Lazarus'[16] emotional theories, while the most representative 
example and the most widely applied one in computer systems is the OCC model by Ortony, Clore and Collins [17].

Sub-cognitive approaches, mainly inspired by the field of neural science, treat emotion as a result of physiological processes that involve issues such as electrical signal transmission and changes in body chemistry. Examples of this category are Damasio [1] and Le Doux's [2] theories.

\section{Designing an emotional agent system}

A hybrid approach, combining ideas from both cognitive and non-cognitive theories of emotion activation was presented in an influential work by Carroll Izard in 1993 [18]. Attempting to bridge the gap between two strong standing, independent pproaches, Izard proposes that emotions cannot be simply treated as something belonging to the realm of either the cognitive or the sub-cognitive, and that the final state and response of an emotional agent is the resultant of a multistage process involving cognitive and non-cognitive functions. Izard argues there are numerous sources of emotion activation, falling into four broad classes: Neural, sensorimotor, motivational and cognitive.

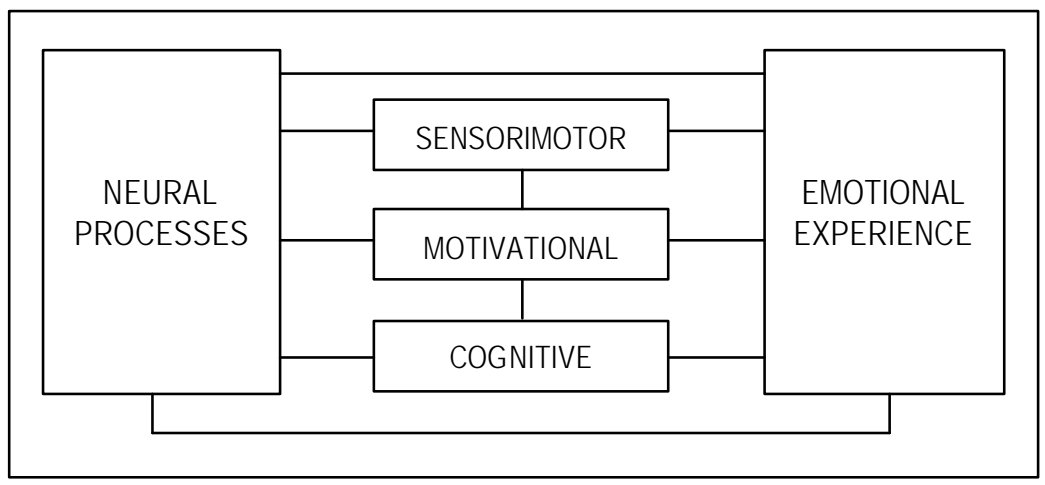

Fig.1: Carroll Izard's four systems of emotion activation (Izard, 1993)

The neural subsystem includes processes such as neural signal transmission, temperature effects, hormone-affected functions or sleep, diet, environmental conditions. The sensorimotor system includes processes such as facial expressions, body posture or activity that can either elicit emotion or affect ongoing emotional experiences. The motivational subsystem includes drives that can lead to emotion generation, such as thirst or hunger, or processes where emotions like sadness can activate others like anger. The cognitive subsystem includes higher-level processes, like appra isal of situations, belief revision or causal attributions.

Although Izard's model does not include implementation details, it illustrates the importance of multiple levels of emotion activation processes, as well as estab- 
lishing a body-mind connection, by attributing emotion elicitation either to cognitive or non-cognitive processes.

\section{Combining Izard's theory with SimHuman}

Adopting Izard's ideas, we are currently working on an emotional agent model we intend to apply over the existing SimHuman architecture. SimHuman, described in [19], is an implemented virtual agent architecture as well as a tool for the creation of virtual agent environments, supporting real-time animation features and incorporating. The SimHuman platform allows users to define and animate three-dimensional scenes with an arbitrary number of objects, virtual agents and user-controlled avatars and has embedded characteristics such as Inverse Kinematics, Physically Based Modeling, Collision Detection and Response, and Vision. SimHuman agents have perception and task control capabilities that can be programmed using SLaVE, a custom high level scripting language [20].

SimHuman's architecture allows the implementation of intelligent virtual agents that are easily adjusted and reprogrammed and can be further connected to more complex AI modules. The agents' functionality is divided into two independent layers with several modules that communicate with each other using certain protocols. The basic layer is the physical layer, which contains the modules that can directly control the agent's body and communicates with the environment through sensing and acting. Above the physical layer there is a cognitive one responsible for higher level functions, such as establishing beliefs through perceptive processes and controlling the agent's behavior using decision or task monitoring and control functions.

Further work on the SimHuman architecture led us to a reconsideration of the initial design. The former labeling of SimHuman's subsystems as cognitive and physical, although useful to distinguish between processes at different levels of abstraction, is misleading when considered with actual human processes in mind. A new approach on a more comprehensive, three-layer architecture has been adopted, distinguis hing between Cognitive and Sub-Cognitive layers, while at the bottom level a Physical layer exists, incorporating the majority of functions performed by the former SimHuman architecture. The initial design is incomplete in respect to what would formally be described as a full-fledged cognitive layer as most of the rational processes in SimHuman are pre-scripted in SLaVE rather than dynamically created. A dynamic action selection and decision making mechanism like a full-scale intelligent planner has to be incorporated as a core component of the Cognitive Layer, so that high-level, deliberative decision-making functions of the agent can be simulated.

Further work was also considered necessary in respect to the reactive control capabilities of the agent, so that lower-level, spontaneous behaviour can be generated according to appropriate stimuli from the environment. The redesign of the initial agent architecture presented in the current work aims towards addressing problems such as the above and providing a unified framework that will incorporate all basic agent functions. 


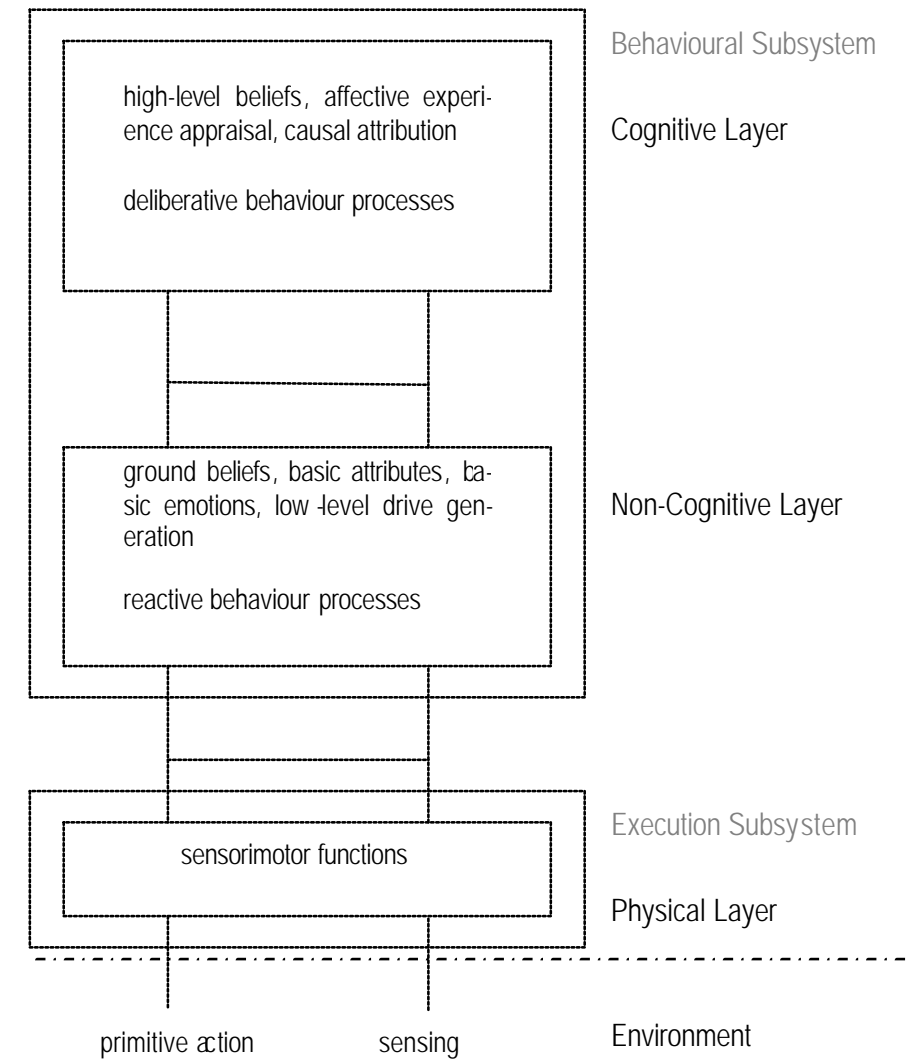

Fig.2: Information flow among system layers

The hack of any emotion-awareness or emotion-handling capabilities, is addressed by the incorporation of an emotional subsystem, inspired by Izard's ideas. In a way similar to works like Cathexis [21], the model designed for the emotional system is a simplification of Izard's four-system model and consists of two generic components, a cognitive and a non-cognitive one. Emotional components in the newly adopted architecture cannot be distinguished as discrete modules, in accordance with the concept of emotion as a necessary ingredient for intelligence. Emotional processes intertwined with deliberative and reactive decision-making processes as parts of the cognitive and the non-cognitive layers, respectively. This approach raises the need for a unified mechanism that can handle emotions along with rational decision making functions. Parallel work is being conducted towards this direction on a continuous planning system taking emotion into account as a necessary element that can affect goal generation and action selection [22].

The new architecture takes into account various constituents of an emotional experience and how that can affect decision making, incorporating basic emotions 
[23], personality models, basic physical, emotional and mental attributes, as well as low and high level drives. The incorporation of these concepts is of major importance, as they are factors that can influence the generation or selection of goals and actions by the agent, but also affect emotion elicitation.

There is substantial interaction between different components of the architecture. As shown in Fig.2,3, process flow in the system does not follow a straightforward in/out execution cycle; several loops might occur in the process, instead. Actions generated in either the Cognitive or the Non-Cognitive Layers can either be extrovert, sending tasks for execution to the Physical Layer, or introvert, actions to be performed by other components. These can either be instructions for further reasoning, or plain information update and communication instructions.

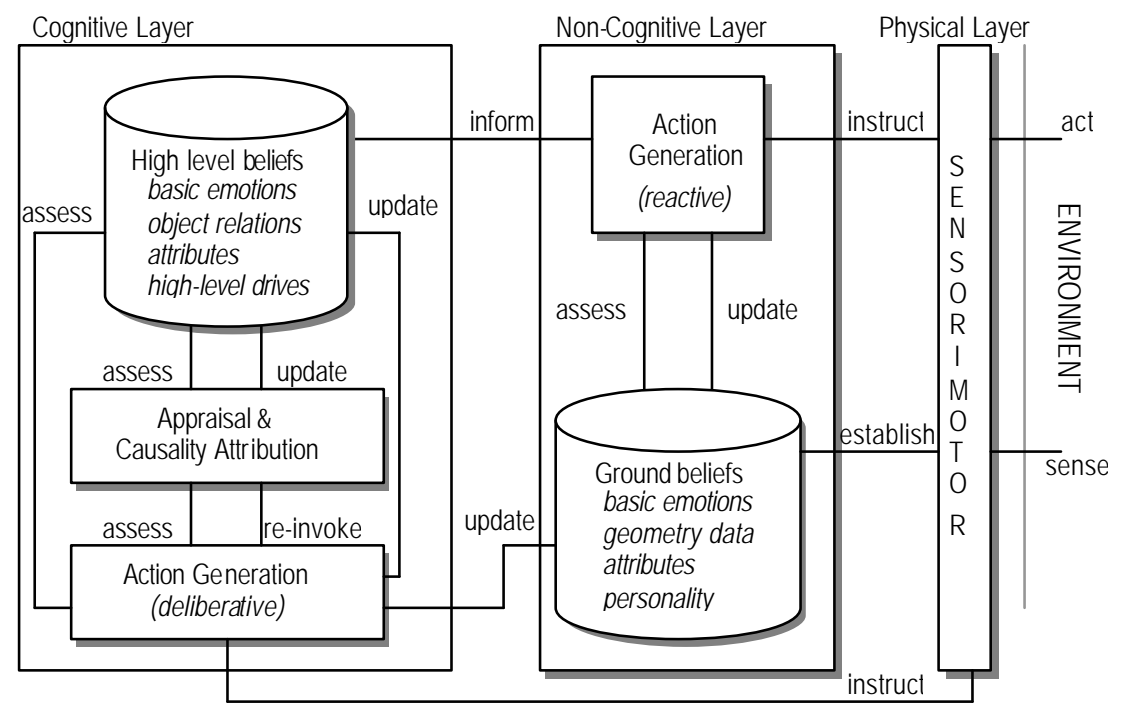

Fig.3: Process interaction

\section{Conclusions}

The importance of emotions as a necessary component in human intelligence has been acknowledged from researchers in various disciplines recently. This implies the necessity of taking emotions into account in every agent architecture targeted towards real-life applications. Such an architecture, intended for application on Intelligent Virtual Environment systems is the one proposed by the authors in this paper. Although at an early stage of development, the authors claim that the approach adopted can adequately support complex reasoning and social awareness capabilities, through the incorporation of an emotion-handling mechanism intertwined with the deliberative and reactive components of the behavioural subsystem of the architecture. 


\section{References}

1. Damasio, A.: Descartes' Error, Gosset/Putnam Press, New York (1994)

2. LeDoux, J.: The Emotional Brain, Simon \& Schuster, New York (1998)

3. Simon, H. A.: Motivational and emotional controls of cognition, in Models of Thought, Yale University Press, (1979) 29-38

4. Sloman, A. and Croucher, M.: Why robots will have emotions, in Proceedings of the Seventh IJCAI, Vancouver, B.C. (1981), 197-202

5. Minsky, M.: The Society of Mind, MIT Press, Cambridge, Massachussets (1985)

6. Elliott, C.: The Affective Reasoner: A Process Model of Emotions in a MultiAgent System. Ph.D. Thesis, The Institute for the Learning Sciences, Northwestern University, 1992.

7. Maes, P., Darrell, T., Blumberg, B., Pentland, A.: The ALIVE System: Wireless, Full-Body Interaction with Autonomous Agents. Multimedia Sy stems 5(2): 105112 (1997)

8. Picard R.: Affective Computing, MIT Press, Cambridge, Massachussets, 1997

9. Reilly W. S., Bates, J.: Building Emotional Agents, CMU-CS-92-143, 1992

10. Luck, M., Aylett, R.: Applying Artificial Intelligence to Virtual Reality: Intelligent Virtual Environments. Applied Artificial Intelligence 14(1): 3-32 (2000)

11. Kleinginna, P. \& Kleinginna, A.: A categorized list of emotion definitions with suggestions for a consensual definition. Motivation and Emotion, 5, 345-379

12. Oatley, K.: Emotions, in the MIT Encyclopedia of Cognitive Sciences, R.A. Wilson, F. Kiel (Eds), MIT Press, Cambridge, Massachussets, 1999

13. Cavazza, M., Charles, F., Mead, S.J.: Character-Based Interactive Storytelling, IEEE Intelligent Systems, July/August 2002, pp 17-24

14. Marsella S., Gratch, J.: Modeling the Interplay of Emotions and Plans in MultiAgent Simulations, in Proceedings of the 23rd Annual Conference of the Cognitive Science Society, Edinburgh, Scotland, 2001

15. Frijda, N.: The Emotions, Cambridge University Press, 1986

16. Lazarus, R.E.: Cognition and motivation in emotion, American Psychologist, 46(4):352-367, 1991

17. Ortony, A., Clore, G., Collins, A.: The Cognitive Structure of Emotions, Cambridge University Press, Cambridge, 1988

18. Izard, C.E.: Four Systems for Emotion Activation: Cognitive and Noncognitive Processes, Psychological Review, 100 (1), pp 68-90.

19. Vosinakis, S., Panayiotopoulos, T.: SimHuman : A Platform for real time Virtual Agents with Planning Capabilities, IVA 2001, 3rd International Workshop on Intelligent Virtual Agents, Madrid, Spain, September 10-11, 2001

20. Vosinakis, S., Panayiotopoulos, T.: Programmable Agent Perception in Intelligent Virtual Environments. IVA 2003: 202-206

21. Velasquez, J. D.: Modeling emotions and other motivations in synthetic agents. In AAAI 97, pages 10-15. ACM 1997.

22. Avradinis, N., Aylett R.: Agents with No Aims: Motivation-Driven Continuous Planning. In: IVA 2003: 269-273

23. Ekman, P.: Basic emotions. In T. Dalgleish and T. Power (Eds.) The Handbook of Cognition and Emotion. Pp. 45-60. John Wiley \& Sons Ltd, Sussex, U.K, (1999).

This work has been funded by the University of Piraeus Research Centre 